\title{
Norwegian GPs' participation in multidisciplinary meetings: A register-based study from 2007
}

\author{
Øystein Hetlevik*, Sturla Gjesdal
}

\begin{abstract}
Background: An increasing number of patients with chronic disorders and a more complex health service demand greater interdisciplinary collaboration in Primary Health Care. The aim of this study was therefore to identify factors related to general practitioners (GPs), their list populations and practice municipalities associated with a high rate of GP participation in multidisciplinary meetings (MDMs).

Methods: A national cross-sectional register-based study of Norwegian general practice was conducted, including data on all GPs in the Regular GP Scheme in 2007 ( $N=3179$ ). GPs were grouped into quartiles based on the annual number of MDMs per patient on their list, and the groups were compared using one-way analysis of variance. Binary logistic regression was used to analyse associations between high rates of participation and characteristics of the GP, their list population and practice municipality.
\end{abstract}

Results: On average, GPs attended 30 MDMs per year. The majority of the meetings concerned patients in the age groups 20-59 years. Psychological disorders were the motivation for 53\% of the meetings. In a multivariate logistic regression model, the following characteristics predicted a high rate of MDM attendance: younger age of the GP, with an OR of 1.6 (95\% Cl 1.2-2.1) for GPs < 45 years, a short patient list, with an OR of 4.9 (3.2-7.5) for list sizes below 800 compared to lists $\geq 1600$, higher proportion of psychological diagnosis in consultations (OR3.4 (2.6-4.4)) and a high MDM proportion with elderly patients (OR 4.1 (3.3-5.4)). Practising in municipalities with less than 10,000 inhabitants (OR 3.7 (2.8-4.9)) and a high proportion of disability pensioners (OR 1.6 (1.2-2.2)) or patients receiving social assistance (OR $2.2(1.7-2.8)$ ) also predicted high rates of meetings.

Conclusions: Psychological problems including substance addiction gave grounds for the majority of MDMs. GPs with a high proportion of consultations with such problems also participated more frequently in MDMs. List size was negatively associated with the rate of MDMs, while a more disadvantaged list population was positively associated. Working in smaller organisational units seemed to facilitate cooperation between different professionals. There may be a generation shift towards more frequent participation in interdisciplinary work among younger GPs.

\section{Background}

Chronic diseases represent $77 \%$ of the disease burden in Europe[1]. Care of patients with longstanding illness is therefore one of the main responsibilities of general practitioners (GPs). Health services have become more complex involving numerous professional groups, which demands more collaborative health and social services [2-5].

Studies have indicated that well-functioning multidisciplinary teamwork gives positive health outcomes for some patient groups [4-8]. Several barriers to the

\footnotetext{
* Correspondence: oystein.hetlevik@isf.uib.no

* Correspondence: oystein.hetlevik@isf.uib.no
Department of Public Health and Primary Health Care, University of Bergen, Kalfarveien 31, N-5018 Bergen, Norway
}

(c) 2010 Hetlevik and Gjesdal; licensee BioMed Central Ltd. This is an Open Access article distributed under the terms of the Creative

development of multidisciplinary collaboration have been identified, and the structure of the team, clearly defined roles and knowledge of each other's responsibilities are crucial for the team to function effectively $[9,10]$. A GP's participation in a multidisciplinary team attending one of his/her patients is regarded as important in providing an appropriate, coordinated service [11]. However, incorporating physicians in multidisciplinary work seems to be a special challenge $[12,13]$.

With the introduction of a national regular GP scheme in Norway in 2001 all inhabitants were given the right to have a GP as their regular doctor [14]. A main aim of the reform was to improve health services for patients with chronic illnesses. In 2007, 3891 GPs 
contracted to municipalities had list sizes of 500 to 2500 patients. Norwegian GPs are mostly self-employed with $85 \%$ working in group practices, organised independently of other local health services. In their regular practices, Norwegian GPs are mainly paid by fee for services provided, but also have a fixed payment per patient on the list, estimated to give one third of GPs' income. A GP can claim a fee from the National Insurance Services when participating in a meeting with other professionals within health or social services as a part of patient treatment for a specified patient. The authorities have gradually increased the reimbursement for attending multidisciplinary meetings (MDMs) to approximately $€ 100$ per hour at present, which is comparable to income per hour in regular practice.

Since 2001, Norwegian patients who requires longterm, coordinated health services are entitled to have an individual plan (IP) according to The Patients' Rights Act [15]. A recommended working method is to set up a multidisciplinary team comprising health and social workers involved in the care of the patient. The patient should also be an active participant in the team. In most cases the work with IP is organised by municipalities, as shown in table 1.

The Norwegian government are planning a reorganisation of the health services, "The Cooperation Reform", based on the assumption that "patients' needs for coordinated services are not being sufficiently met" [16] The health authorities are also concerned regarding GPs' involvement in multidisciplinary activities [16], based on evaluation reports of the health services, especially of the psychiatric services [17]. List size reduction is proposed as a main tool to improve GPs participation in multidisciplinary teams, without scientific evidence supporting this strategy [16].

The present study explored Norwegian GPs' participation in MDMs concerning their patients, and the health problems addressed. We wanted to investigate the widespread opinion that the frequency of GPs' participation in MDMs is generally low, and that the list size, an indicator of workload, explains possible differences.

The aim of the study was to identify the impact of characteristics of GPs, patient list populations and the practice municipalities on the frequency of the GPs' participation in MDMs.

\section{Methods}

Material

A national cross-sectional register-based study of Norwegian general practice was conducted. All Norwegian GPs participating in the Regular GP Scheme who had practised during the whole year $2007(\mathrm{~N}=3179)$ were included, with a total of 97,091 reported MDMs.

The data were obtained from the following three data sources:

From the national GP register we obtained information on the GP's age and gender, the practice municipality, information on list length and changes in list length, and the age and gender of the patients on the GP's list.

The national research database "FD trygd" contains data on income, education, and receipt of social security and social assistance benefits on all inhabitants. Based on the unique ID number for each person in the national GP register, Statistics Norway linked this information anonymously to all persons on the patient lists $(\mathrm{N}=3,936,126)$.

\section{Table 1 Individual plan - "The Norwegian model" for cooperation in Primary health care}

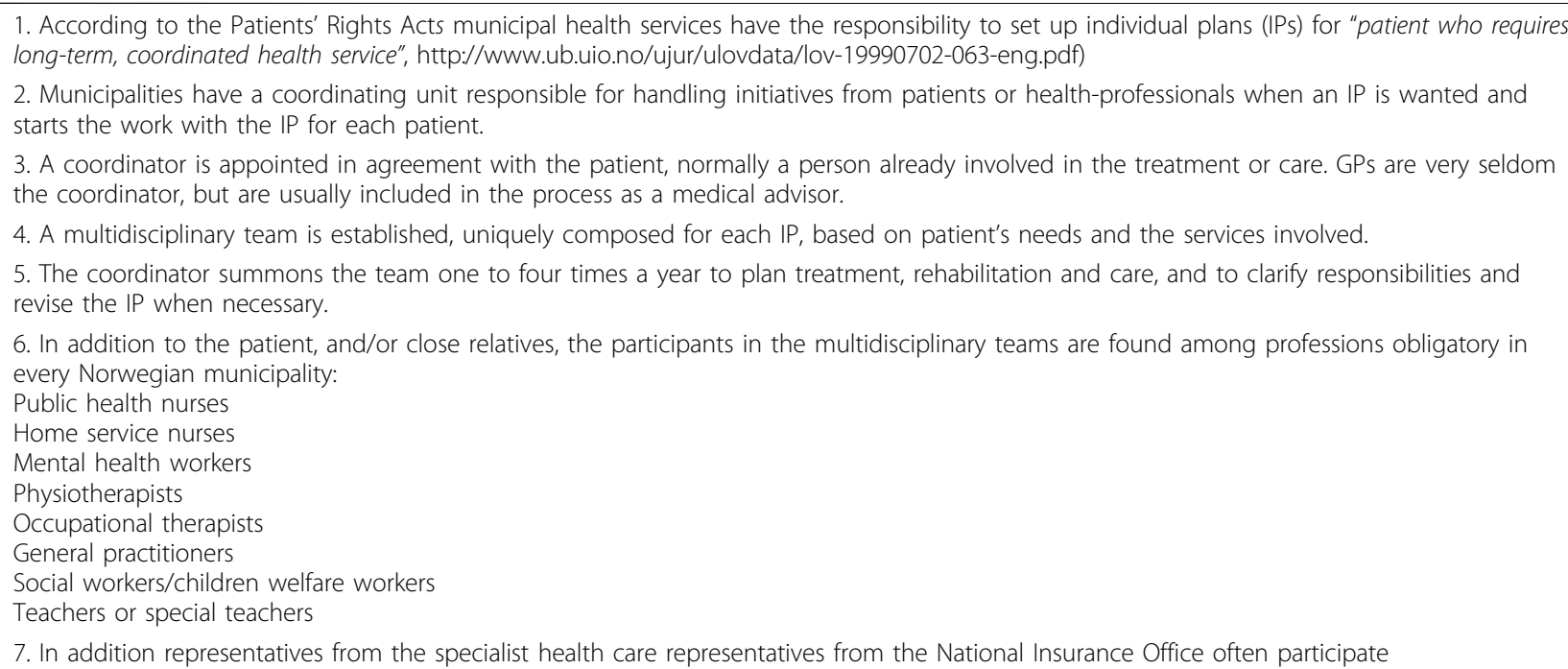

The work with IP are more fully described in documents from the Norwegian Directorate of Health, found at: http://www.helsedirektoratet.no/vp/multimedia/ archive/00010/IS-1292_E_10745a.pdf 
The Norwegian National Insurance Services receives bills from GPs in the fee-for-service system. We obtained anonymised data on all bills from GPs concerning all patient-related activities including participation in MDMs. These bills include a GP identity, the patient age and gender, but no person identification. They also include information of the time used in the meetings and a diagnosis set by the GP according to the International Classification of Primary Health Care (ICPC) [18].

The study was approved by the Norwegian Data Inspectorate and the owners of the databases.

\section{Outcome variable}

We estimated the annual rate of MDMs per patient on the GP's list. Based on this rate, GPs were divided into quartiles, and these groups were compared with respect to GP age and gender, list size, and sociodemographic characteristics of the patient list population. The annual rate of MDMs was dichotomised, defining the 25\% of GPs with the highest annual rates as a "high rate group". This was used as the outcome variable in the logistic regression analyses.

\section{Explanatory variables}

GPs were characterised by age and gender.

The lists were characterised by the number of patients and whether or not the list was open to new patients.

The list populations were characterised by the proportion of males, mean age in the list population, educational level, mean annual income, the proportion of disability pensioners, and the proportion of recipients of social assistance on the list. Educational level was measured by the proportion of patients in the list aged over 20 years with only basic education ( $\leq 9$ years' education). Income level was measured as the mean annual income among those aged 20 and above.

The GP practice municipalities were grouped according to the number of inhabitants.
A GP practice profile was indicated by the proportion of all the GP's ordinary consultations that had a main diagnosis of a psychological problem according to the Pchapter in ICPC.

The MDM proportion with patients $\geq 67$ years adjusted for differences in list population was estimated, and defined as "high" when the proportion of MDMs concerning a patient $\geq 67$ years was equal to or higher than the proportion of list population $\geq 67$ years.

\section{Statistics}

One-way analysis of variance was used to compare the quartiles of GPs according to rates of MDMs.

Binary logistic regression was used to analyse associations between "high" rates of participation in multidisciplinary meetings and GP, list-population and municipality characteristics. First, univariate odds ratios were estimated for all variables; then three multivariate models were constructed by including gradually new sets of variables. There was no reason to assume a linear association between variables and outcome, so variables were categorised by dividing the GPs in three equal sized groups.

The statistical package STATA 11 was used for the analyses.

\section{Results}

On average, GPs attended 30 MDMs a year. The majority of the meetings concerned patients in the age groups 20-59 years (Table 2), and the proportion of male and female patients was almost identical. When the patient was $\geq 70$ years, $75 \%$ of meetings lasted less than 30 minutes, compared to $30 \%$ for patients $<70$ years (not tabled).

Patients with psychological diagnoses were the focus of the majority of the meetings. In $12 \%$ of all meetings a diagnosis of drug or alcohol addiction was used, in $10 \%$ depression and in $10 \%$ a serious mental disorder.

Table 2 Distribution of multidisciplinary meetings attended by 3179 Norwegian GPs in 2007, according to diagnoses ${ }^{1}$ and patient age

\begin{tabular}{lllllll}
\hline & \multicolumn{5}{c}{ Number of meetings (\%) within different age groups } \\
\cline { 2 - 6 } ICPC chapters: $^{1}$ & All ages & $\mathbf{0 - 1 9}$ years & $\mathbf{2 0 - 3 9}$ years & $\mathbf{4 0 - 5 9}$ years & $\mathbf{6 0 - 7 9}$ years & $\mathbf{2 8 0}$ years \\
\hline A - General and unspecified & $10187(10.5)$ & $1223(11.2)$ & $3663(11.3)$ & $3393(10.6)$ & $798(7.3)$ & $1101(10.4)$ \\
K - Cardiovascular & $4903(5.1)$ & $81(0.7)$ & $235(0.7)$ & $953(3.0)$ & $1393(12.7)$ & $2241(21.1)$ \\
L - Musculoskeletal & $11937(12.3)$ & $446(4.1)$ & $3048(9.4)$ & $5789(18.1)$ & $1528(13.9)$ & $1126(10.6)$ \\
N - Neurological & $5528(5.7)$ & $1098(10.0)$ & $1412(4.4)$ & $1696(5.3)$ & $936(8.5)$ & $386(3.6)$ \\
P - Psychological & $52113(53.7)$ & $6451(58.6)$ & $21835(67.3)$ & $17497(54.6)$ & $3771(34.4)$ & $2559(24.1)$ \\
All other diagnoses & $12423(12.7)$ & $1702(15.4)$ & $2248(6.9)$ & $2716(8.4)$ & $2550(23.2)$ & $3206(30.2)$ \\
\hline Sum & $97091(100)$ & $11011(100)$ & $32441(100)$ & $32044(100)$ & $10976(100)$ & $10619(100)$ \\
\hline
\end{tabular}

${ }^{1}$ Diagnosis based on The International Classification of Primary Care [ICPC] 
Although the rates of MDMs clearly fell with larger list size (Figure 1), the mean annual number of meetings varied little between list size groups (min-max: 27-34).

Table 3 presents characteristics of the GPs, divided in quartiles according to rates of MDMs. The rate of meetings increased when the list had a higher proportion of list patients with low education, disability pensioners or social assistance receivers. There were only minor differences between groups in the distribution of diagnoses used in MDMs (not shown).

The univariate odds for being among the quartile of GPs with the highest rate of MDMs increased markedly with decreasing list size (Table 4). GPs with a list size of less than 800 patients had a 7.8 times higher unadjusted odds of being within the high rate group compared to GPs with a list size $\geq 1600$ patients. Male GPs and younger age of the GP were also associated with a high rate of MDMs. There was an association between a high rate of meetings and lower socioeconomic level in the list population when measured by mean annual income or proportion of patients with a low educational level. The proportion of patients on disability pension or receiving social assistance benefits was also associated with high rates of MDMs. The rate of meetings was inversely associated with number of inhabitants in the practice municipality.

In the first multivariate model, list size was adjusted for GP age and gender, and some practice characteristics, with an increase in the effect of list size.

In the second model, list size was also adjusted for list population variables, giving a markedly decrease in the list length effect. In this model GP gender showed no association with high rates of MDMs and the effect of several sociodemographic list population variables decreased or was eliminated.

When adjusting for all GP-, list-, population- and municipality characteristics, a smaller list size remained by far the strongest predictor of a high rate of MDM participation. However, a number of other variables were also shown to increase the odds for a high rate of MDMs: young GP age, a relatively high educational level in the list population, and a high proportion of disability pensioners and recipients of social assistance in the list. A higher proportion of ordinary GP consultations with a psychological diagnosis were associated with a high rate of MDMs. There was also a clear association between high MDM proportion concerning elderly and a high rate of meetings in general. Practising in municipalities with less than

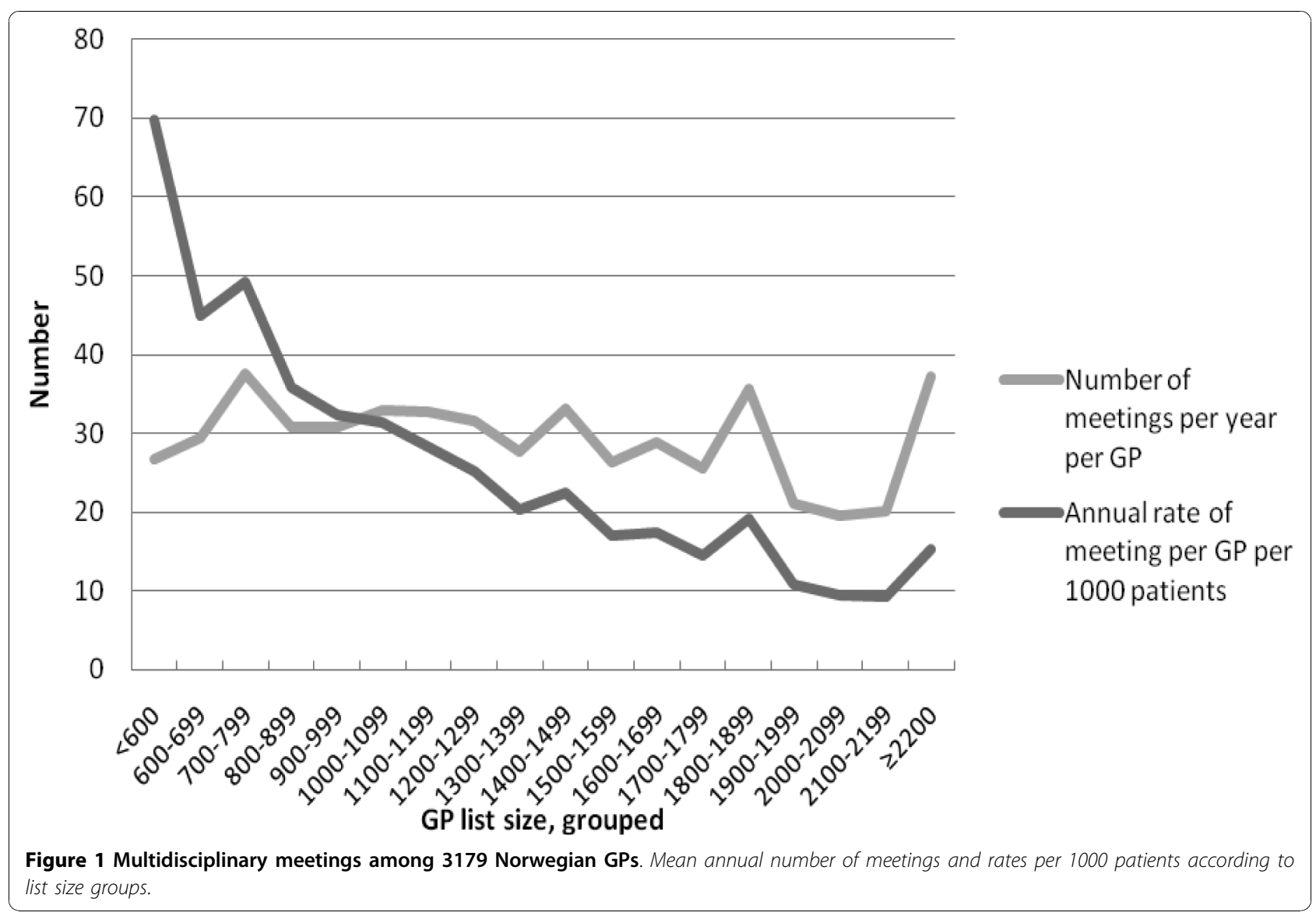


Table 3 GP, list, and list population characteristics among Norwegian GPs, grouped according to frequency of attendance in multidisciplinary meetings (MDMs) in 2007

\begin{tabular}{|c|c|c|c|c|c|}
\hline & & Frequency of & dance in $\mathrm{MDMs}^{1}$ & & \\
\hline & Low & Medium/low & Medium/high & High & p-value \\
\hline Number of GPs & 795 & 795 & 795 & 794 & \\
\hline $\begin{array}{l}\text { Mean annual rate per } 1000 \text { patients } \\
\text { (min-max) }\end{array}$ & $\begin{array}{c}4 \\
(0-7)^{2}\end{array}$ & $\begin{array}{c}12 \\
(7-17)\end{array}$ & $\begin{array}{c}24 \\
(17-35)\end{array}$ & $\begin{array}{c}54 \\
(35-170)\end{array}$ & \\
\hline GP characteristics: & & & & & \\
\hline GP age, mean & 52.2 & 50.0 & 48.5 & 47.3 & $<0.001$ \\
\hline GP gender: percent male & 70.5 & 69.3 & 68.2 & 74.7 & 0.026 \\
\hline GP practice characteristics: & & & & & \\
\hline Consultations with psychological diagnosis ${ }^{3}, \%$ & 8.6 & 9.7 & 10.5 & 11.8 & $<0.001$ \\
\hline Rate MDMs, patients $\geq 67$ years $^{4}$ & 0.6 & 0.8 & 1.0 & 1.6 & $<0.001$ \\
\hline List characteristics: & & & & & \\
\hline Mean list size & 1367 & 1283 & 1225 & 1077 & $<0.001$ \\
\hline Percentage of lists open to new patients & 32.7 & 28.6 & 26.9 & 37.0 & $<0.001$ \\
\hline List population characteristics: & & & & & \\
\hline Persons $\geq 67$ years on the list, $\%$ & 12.9 & 12.3 & 12.1 & 13.0 & 0.014 \\
\hline Men in the list, \% & 49.4 & 49.2 & 49.2 & 51.1 & $<0.001$ \\
\hline Education $\leq 9$ years ${ }^{5}, \%$ & 14.1 & 14.4 & 14.6 & 15.9 & $<0.001$ \\
\hline Mean annual income (NOK1000) & 266 & 248 & 241 & 226 & $<0.001$ \\
\hline Disability pensioners ${ }^{6}, \%$ & 9.5 & 10.1 & 10.7 & 11.7 & $<0.001$ \\
\hline Social assistance recipients ${ }^{7}, \%$ & 3.6 & 4.1 & 4.6 & 5.6 & $<0.001$ \\
\hline
\end{tabular}

One-way analyses of variance. $\mathrm{N}=3179 \mathrm{GPs}$

1) GPs grouped in quartiles based on annual rate of multidisciplinary meetings per patient on the list

2) 83 GPs with no registered meetings

3) Proportion of all consultations in the GPs practices

4) Rate MDMs, patients $\geq 67$ years = Proportion of MDMs concerning a patient $\geq 67$ years/proportion of list population $\geq 67$ years.

5) Proportion among patients $\geq 20$ years

6) Proportion among patients 20-67 years

7) Proportion among patients $20-40$ years

10,000 inhabitants also predicted high rates of meetings when all other variables were adjusted for.

We also made a regression model including information of the GP being a specialist in family medicine or not, and length of practice time in the municipality, without revealing any significant associations with MDM rates (data not shown).

\section{Discussion}

\section{Main findings}

This register-based study on Norwegian GPs' participation in MDMs showed large differences between doctors. On average, GPs participated in one meeting every two weeks. Patients with a psychological diagnosis motivated the majority of the meetings.

The main predictors of a high rate of participation were a short or average list size and practising in a smaller municipality.

A high rate of MDMs was also associated with younger GPs, higher proportion of psychological diagnosis in ordinary GP consultations, higher MDM proportion concerning elderly patients and markers of lower socioeconomic status in the list population, except for education.

The outcome variable, rates of multidisciplinary meetings We used rates of participation in MDMs per patient on the list as the outcome measure. From a patient perspective, this is an indicator of the probability that the patient's GP will participate in multidisciplinary teams if necessary. Studies have indicated that GP participation in such collaboration is advantageous for patients [7]. Engaging physicians in multidisciplinary work has been shown to be a challenge, and there is a demand to increase GPs' participation based on possible health gain, perspectives of patient rights and political wishes $[6,12,16,19,20]$. One could argue that participating in multidisciplinary meetings is advantageous for patients with chronic diseases, based on findings in earlier studies [6-8,21,22]. Thus, the MDM rate is a measure of GP's adherence to "best practice" according to the Government's policy/guidelines for collaboration. 
Table 4 List size and characteristics of GPs, list populations and practice municipalities associated with a high rate ${ }^{1}$ of multidisciplinary meetings (MDMs) in Norwegian general practice

\begin{tabular}{|c|c|c|c|c|c|c|c|c|c|c|c|c|c|}
\hline & \multirow[b]{2}{*}{$N$} & \multicolumn{3}{|c|}{ Univariate analyses } & \multicolumn{3}{|c|}{ Model 1} & \multicolumn{3}{|c|}{ Model 2} & \multicolumn{3}{|c|}{ Model 3} \\
\hline & & $O R$ & $95 \% \mathrm{Cl}$ & $p$-value & $O R$ & $95 \% \mathrm{Cl}$ & $p$-value & OR & $95 \% \mathrm{Cl}$ & $p$-value & $O R$ & $95 \% \mathrm{Cl}$ & $p$-value \\
\hline \multicolumn{14}{|l|}{ List size } \\
\hline$\geq 1600$ & 476 & Ref & & & Ref & & & Ref & & & Ref & & \\
\hline $1200-1599$ & 1162 & 2.3 & $1.7-3.3$ & $<0.001$ & 2.6 & $1.8-3.7$ & $<0.001$ & 2.3 & $1.6-3.3$ & $<0.001$ & 2.3 & $1.6-3.3$ & $<0.001$ \\
\hline $800-1199$ & 1143 & 4.1 & $3.0-5.7$ & $<0.001$ & 4.9 & $3.4-6.9$ & $<0.001$ & 3.6 & $2.5-5.1$ & $<0.001$ & 2.9 & $2.0-4.2$ & $<0.001$ \\
\hline$<800$ & 398 & 7.8 & $5.4-11.3$ & $<0.001$ & 10.7 & $7.2-15.8$ & $<0.001$ & 7.1 & $4.7-10.7$ & $<0.001$ & 4.9 & $3.2-7.5$ & $<0.001$ \\
\hline \multicolumn{14}{|l|}{ GP age } \\
\hline$\geq 55$ years & 1014 & Ref & & & Ref & & & Ref & & & Ref & & \\
\hline $45-54$ years & 1235 & 1.2 & $0.9-1.4$ & 0.17 & 1.2 & $1.0-1.6$ & 0.071 & 1.3 & $1.1-1.6$ & 0.020 & 1.3 & $1.0-1.6$ & 0.058 \\
\hline$<45$ years & 930 & 2.1 & $1.7-2.6$ & $<0.001$ & 1.9 & $1.5-2.5$ & $<0.001$ & 1.9 & $1.5-2.4$ & $<0.001$ & 1.6 & $1.2-2.1$ & $<0.001$ \\
\hline \multicolumn{14}{|l|}{ GP gender } \\
\hline Female & 932 & Ref & & & Ref & & & Ref & & & Ref & & \\
\hline Male & 2247 & 1.3 & $1.1-1.6$ & 0.004 & 2.0 & $1.6-2.4$ & $<0.001$ & 1.2 & $0.9-1.7$ & 0.21 & 1.3 & $1.0-1.9$ & 0.076 \\
\hline \multicolumn{14}{|c|}{ MDM proportion with patients $\geq 67$} \\
\hline Low $^{2}$ & 2225 & Ref & & & Ref & & & Ref & & & Ref & & \\
\hline $\mathrm{High}^{3}$ & 954 & 3.3 & 2.8-3.9 & $<0.001$ & 3.8 & $3.2-4.6$ & $<0.001$ & 4.4 & 3.6-5.3 & $<0.001$ & 4.1 & $3.3-5.0$ & $<0.001$ \\
\hline
\end{tabular}

Proportion of consultations with a psychological diagnosis*

\begin{tabular}{|c|c|c|c|c|c|c|c|c|c|c|c|c|c|}
\hline Low $(<8 \%)$ & 1060 & Ref & & & Ref & & & Ref & & & Ref & & \\
\hline Medium (8-11\%) & 1060 & 1.1 & $0.9-1.4$ & 0.33 & 1.3 & $1.0-1.6$ & 0.05 & 1.2 & $1.0-1.6$ & 0.11 & 1.4 & $1.1-1.8$ & 0.010 \\
\hline High (> 11\%) & 1059 & 2.4 & $2.0-2.9$ & $<0.001$ & 2.8 & $2.2-3.4$ & $<0.001$ & 2.7 & $2.1-3.4$ & $<0.001$ & 3.4 & $2.6-4.4$ & $<0.001$ \\
\hline \multicolumn{14}{|c|}{ Proportion $\geq 67$ years* } \\
\hline Low $(<9 \%)$ & 1060 & Ref & & & & & & Ref & & & Ref & & \\
\hline Medium (9-15\%) & 1060 & 1.0 & $0.9-1.3$ & 0.76 & & & & 1.0 & $0.8-1.3$ & 0.94 & 0.9 & $0.7-1.2$ & 0.35 \\
\hline High (> 15\%) & 1059 & 1.2 & $1.0-1.5$ & 0.056 & & & & 1.0 & $0.8-1.4$ & 0.80 & 0.9 & $0.6-1.2$ & 0.39 \\
\hline \multicolumn{14}{|c|}{ Proportion male patients* } \\
\hline Low $(<48 \%)$ & 1060 & Ref & & & & & & Ref & & & Ref & & \\
\hline Medium (48-54\%) & 1060 & 1.5 & $1.2-1.9$ & $<0.001$ & & & & 1.6 & $1.2-2.3$ & 0.002 & 1.3 & $1.0-1.9$ & 0.076 \\
\hline High (> 54\%) & 1059 & 1.6 & $1.3-2.0$ & $<0.001$ & & & & 1.9 & $1.4-2.7$ & $<0.001$ & 1.6 & $1.2-2.3$ & 0.005 \\
\hline \multicolumn{14}{|c|}{ Proportion with education $\leq 9$ years ${ }^{4}{ }^{*}$} \\
\hline Low $(<12 \%)$ & 1060 & Ref & & & & & & Ref & & & Ref & & \\
\hline Medium (12-17\%) & 1060 & 1.0 & $0.9-1.3$ & 0.71 & & & & 0.7 & $0.5-0.8$ & 0.001 & 0.6 & $0.4-0.8$ & $<0.001$ \\
\hline High (> 17\%) & 1059 & 1.7 & $1.4-2.1$ & $<0.001$ & & & & 0.7 & $0.5-1.1$ & 0.089 & 0.6 & $0.4-0.9$ & 0.012 \\
\hline \multicolumn{14}{|c|}{ Mean annual income among patients ${ }^{4}$} \\
\hline$<210,000$ n.kr & 799 & Ref & & & & & & Ref & & & Ref & & \\
\hline $210000-250000$ & 1283 & 0.7 & $0.6-0.8$ & $<0.001$ & & & & 0.8 & $0.6-1.0$ & 0.046 & 0.9 & $0.7-1.2$ & 0.40 \\
\hline$>250000$ & 1097 & 0.3 & $0.3-0.4$ & $<0.001$ & & & & 0.5 & $0.3-0.6$ & $<0.001$ & 0.7 & $0.5-0.9$ & 0.008 \\
\hline \multicolumn{14}{|c|}{ Proportion disability pensioners ${ }^{5 *}$} \\
\hline Low $(<8 \%)$ & 1060 & Ref & & & & & & Ref & & & Ref & & \\
\hline Medium (8-11\%) & 1060 & 1.5 & $1.3-1.9$ & $<0.001$ & & & & 1.7 & $1.3-2.2$ & $<0.001$ & 1.6 & $1.3-2.1$ & $<0.001$ \\
\hline High (> 12\%) & 1059 & 1.9 & $1.6-2.4$ & $<0.001$ & & & & 1.7 & $1.2-2.6$ & 0.001 & 1.6 & $1.2-2.2$ & 0.005 \\
\hline \multicolumn{14}{|c|}{ Proportion with social assistance ${ }^{6 *}$} \\
\hline Low $(<3 \%)$ & 1060 & Ref & & & & & & Ref & & & Ref & & \\
\hline Medium (3-5\%) & 1060 & 1.4 & $1.1-1.8$ & 0.002 & & & & 1.1 & $0.9-1.4$ & 0.37 & 1.2 & $0.9-1.6$ & 0.14 \\
\hline High (> 5\%) & 1059 & 3.0 & $2.5-3.7$ & $<0.001$ & & & & 1.9 & $1.5-2.4$ & $<0.001$ & 2.2 & $1.7-2.8$ & $<0.001$ \\
\hline \multicolumn{14}{|c|}{ Inhabitants in practice municipality } \\
\hline$>50,000$ & 1103 & Ref & & & & & & & & & Ref & & \\
\hline $20-50,000$ & 667 & 0.9 & $0.7-1.2$ & 0.37 & & & & & & & 0.9 & $0.7-1.2$ & 0.43 \\
\hline $10-20,000$ & 514 & 1.7 & $1.3-2.2$ & $<0.001$ & & & & & & & 1.5 & $1.1-2.1$ & 0.005 \\
\hline
\end{tabular}


Table 4 List size and characteristics of GPs, list populations and practice municipalities associated with a high rate ${ }^{1}$ of multidisciplinary meetings (MDMs) in Norwegian general practice (Continued)

$<10,000 \quad 895 \quad 4.4 \quad 3.6-5.5<0.001$
Binary logistic regression analyses. N = $3179 \mathrm{GPs}$
1) "High rate" defined as $>35$ meetings per 1000 patients per year
2) Proportion of MDMs concerning patients $\geq 67$ years lower than the proportion of list population $\geq 67$ years
3) Proportion of MDMs concerning patients $\geq 67$ years equal to or higher than the proportion of list population $\geq 67$ years
4) Among list population $\geq 20$ years
5) Proportion disability pensioners in the age group $20-67$ years
6) Proportion with social assistance in the age group $20-40$ years
*) Categorised variables with three equal sized groups of GPs, percentages are give, rounded to nearest whole numbers, to illustrate differences between GP
groups.

The rate of MDMs does not show the total amount of a GP's cooperation, which is also conducted by telephone or by written communication. However, meetings have been shown to be a facilitator of teamwork [10].

\section{GP variables}

The study showed that younger GPs participated more frequently in MDMs. This could indicate a generation shift among Norwegian GPs, towards younger GPs working more closely with other professional groups, perhaps as a result of more emphasis on teamwork in the medical curriculum.

\section{The effect of list size}

With the introduction of the regular GP Scheme, a list size of 1200-1500 patients was estimated to give a reasonable workload within the Norwegian PHC, were GPs are obliged to work one day a week in municipality health service for children or elderly. Workload is shown to increase with increasing list sizes [23,24]. GPs with larger list sizes have to prioritise more strongly in his or her work, and according to our findings, attendance in meetings may be given lower priority. It has been shown that it is necessary to allocate time to implement multidisciplinary teamwork [25], and that a high workload influences practice performance according to measures of quality of care $[24,26]$.

On the other hand, teamwork may be a way of sharing responsibility for patient treatment. GPs have a central role in continuous care, as stated in the regulations of the GP scheme, and active cooperation with other health and social workers in patient treatment and care could ease GP workload and responsibility [12]. This perspective could motivate GPs to participate in team collaboration. However, this is not always included in the assessment when prioritising.

Although large list size seems to predict lower participation in MDMs, it is not obvious that reducing list sizes, as proposed by the Norwegian authorities, would change the GPs' practice. The ability to tackle a large list and the extent of collaboration may have a common explanation as a part of GPs' attitudes or practice styles shown to be
GP-dependent in respect to other practice factors such as professional competence and time use [27]. However, this not possible to assess with register data

\section{Socioeconomic status}

Low socioeconomic status in a population increases the prevalence of chronic diseases [28,29], and probably also the need for multidisciplinary coordinated services. When we adjusted for other variables in our model, the predictive value of low income among the list population was reduced, and low educational level became inversely associated with rate of MDMs. These variables are indicators of the disease burden in the total list population. Consequently list populations with a higher average socioeconomic level might have a lower disease burden, and the GPs may more easily engage in teamwork for the patients with special needs.

Being a disability pensioner or receiving social assistance implies a risk of serious health problems and also the need for an IP [30,31]. This study shows that the proportion of a list population dependent on state income supplements is a stronger predictor of a GP's participation in MDMs compared to socioeconomic variables aggregated at list population level.

\section{Psychological diagnoses}

The motivation for most meetings was patients with psychological diagnoses. In this field, teamwork has been shown to be efficient in improving the outcome and quality of patient treatment $[7,21,32]$. Over the last decade, $\mathrm{PHC}$ has been given more responsibility in the treatment of patients with psychiatric diseases, and new groups of health workers are involved in both PHC and specialised care, resulting in more complex services. The high number of meetings within the field of mental health indicates that Norwegian GPs have responded to this challenge, and take an active role in teamwork concerning patients with mental health problems including drug and alcohol dependence. The study showed a strong association between a high proportion of ordinary consultations with psychological diagnoses and a high participation rate in MDMs. This can reveal 
differences in the mental health of the list population, but is probably also dependent on the GPs' field of interest.

\section{Elderly patients}

The burden of disease is higher among elderly people, with an increasing need for coordinated health services, and a multidisciplinary approach has been shown to improve health outcome among the elderly [22]. The proportion of all the GPs' MDMs concerning elderly patients is relatively low, however, and does not reflect this need. IP is seldom used as a tool for cooperation among the elderly. Probably other channels, for instance within home nursing services and in nursing homes, are used in the collaboration for this patient group, without systematically involving the GPs.

A high proportion of elderly in the list had no impact on the general rate of participation in MDMs. Having a high MDM proportion with elderly patients was clearly associated with high rates of MDMs generally. Meetings concerning elderly patients were also generally shorter. These findings indicate local variation in cooperative routines for the elderly, where some GPs seems to be participating more frequently but in shorter meetings concerning elderly. Such local variations may explain a part of the large differences in MDM rates between GPs in general. Including more teamwork concerning elderly patients into practice routines is probably desirable and may contribute to higher rates of MDMs.

\section{Small municipalities}

The association between high collaborative activity and practising in the smallest municipalities can be explained by the geographic and organisational nearness of services seen in these municipalities. These structural factors are not directly transferable to larger municipalities, but suggest that organising PHC based on a smaller geographical and organisational units, could improve multidisciplinary cooperation. Affiliating other health or social workers to GP group practices, as seen in the UK [33], may to some extent simulate a small municipality health service and could improve interdisciplinary cooperation.

\section{Strengths and Limitations}

This study included all Norwegian GPs practising during the entire year 2007 and was based on register data for a full calendar year. This eliminates any selection bias of GPs' responses being influenced by his/her interest in collaboration or research in PHC.

The bills reporting meetings are reliable markers of this activity because the fee constitutes the sole payment and reporting is therefore probably complete. Over- reporting of meetings constitutes a criminal act, and is not very common.

Coding according to ICPC has been shown to be valid at chapter level and is assumed to reflect the actual health problems motivating the meetings $[34,35]$.

A major limitation with our register data is the lack of information on the specific purpose of meetings and which other services were involved. We assume the majority of the meetings to be part of work with IP, in multidisciplinary teams, since this is the main organisational model for cooperation in PHC. However, GPs also participate in ad hoc meetings for patients in the need of coordinated services, when an IP is not established.

Because the GP bills were anonymised we did not know the number of different patients involved in the MDMs, but only the number of meetings, and age and gender of the patients involved.

Another weakness of the study is that no information was available on the number of patients with severe chronic disease in the list populations. We used sociodemographic indicators as a measure of health needs at list population level $[29,36]$. These aggregated indicators do not necessarily reflect diversities in the number of patients in need of multidisciplinary cooperation. The prevalence of mental health problems in the GPs' list populations was indicated by the proportion of ordinary GP consultations that was diagnosed with an ICPC diagnosis indicating psychological symptoms or disorders as the reason for the consultation.

We had no data on the GPs' skills or training in multidisciplinary work, attitudes to collaboration or the quality of the teamwork. Studies with a different design are necessary to explore such qualitative aspects. There was no information available about the organisation of practices, such as single-handed or group practice and the number of staff affiliated.

Finally, logistic regression analyses in cross-sectional samples reveal associations, and interpretation of causality must be made with caution.

\section{Further research}

There is a need for more knowledge of the possible health effects of the Norwegian cooperation model based on IPs and multidisciplinary meetings. It is especially important to assess whether multidisciplinary team meetings are used for the patients with the highest needs for health service. The usefulness for patients of involving the GPs in MDMs should be studied, preferable in randomised controlled trials, which however seldom is feasible. Why younger GPs participate more in MDMs should also be studied, because this knowledge may be useful when designing educational programs for GPs in this area. 


\section{Conclusion}

Norwegian GPs have substantial engagement in multidisciplinary patient-centred meetings, with an average of one meeting every two weeks. However, rates of MDMs vary considerably between GPs. The present study identified several structural and GP related factors that influence GPs' multidisciplinary cooperation. These findings should be considered when policy changes for general practice are developed.

Positive health outcomes for patients with functioning multidisciplinary cooperation in their treatment and care have been documented. However, the benefit of teams seems dependent on structure and procedures $[4,5,8,10,37,38]$.

\section{Acknowledgements}

The study was funded by the "Fund for research in General Practice, the Norwegian Medical Association"

\section{Authors' contributions}

Both $\varnothing \mathrm{H}$ and SG have contributed to the statistical analyses, to writing the manuscript and have approved the final version.

\section{Competing interests}

The authors declare that they have no competing interests.

Received: 3 March 2010 Accepted: 15 November 2010

Published: 15 November 2010

\section{References}

1. World Health Organisation: Preventing chronic disease: a vital investment. Geneva; 2005.

2. Davies $C:$ Getting health professionals to work together. $B M J$ 320:1021-1022.

3. Stange KC: The problem of fragmentation and the need for integrative solutions. Ann Fam Med 2009, 7:100-103.

4. Nolte E, McKee M, (Eds): Caring for people with chronic conditions - A health system perspective. Open University Press; 2008.

5. Oxman AB, Flottorp A, Lewin SA, Lindahl SAK: Integrated Health Care for People with Chronic Conditions. Oslo: Norwegian Knowledge Centre for the Health Services; 2008

6. Bodenheimer T, Wagner EH, Grumbach K: Improving primary care for patients with chronic illness. JAMA 2002, 288:1775-1779.

7. Hviding K, Bugge P, Ekern P, Brelin P, Høifødt T, Nessa J, Flottorp S: Collaborative care initiatives for patients with serious mental disorders treated in primary care setting. Systematic review of the evidence. Oslo: Norwegian Knowledge Centre for the Health Services; 2008

8. Zwarenstein M, Goldman J, Reeves S: Interprofessional collaboration: effects of practice-based interventions on professional practice and healthcare outcomes. Cochrane database of systematic reviews (Online) 2009, CD000072.

9. Murray S, Silver I, Patel D, Dupuis M, Hayes SM, Davis D: Community group practices in Canada: are they ready to reform their practice? J Contin Educ Health Prof 2008, 28:73-78.

10. Sargeant J, Loney E, Murphy G: Effective interprofessional teams: "contact is not enough" to build a team. J Contin Educ Health Prof 2008, 28:228-234.

11. Stille CJ, Jerant A, Bell D, Meltzer D, Elmore JG: Coordinating care across diseases, settings, and clinicians: a key role for the generalist in practice. Ann Intern Med 2005, 142:700-708.

12. Hansson A, Friberg F, Segesten K, Gedda B, Mattsson B: Two sides of the coin - general practitioners' experience of working in multidisciplinary teams. J Interprof Care 2008, 22:5-16.
13. Wright B, Lockyer J, Fidler H, Hofmeister M: Roles and responsibilities of family physicians on geriatric health care teams: Health care team members' perspectives. Can Fam Physician 2007, 53:1954-1955.

14. The Norwegian Ministry of Health and Social Affairs: Regulation relating to a Municipal Regular GP Scheme. Oslo; 2000 [http://www.regjeringen.no/ en/dep/hod/dok/lover_regler/reglement/2000/regulation-relating-to-amunicipal-regul.html?id=420530].

15. Norwegian Directorate of Health: Individual Plan - A folder. Oslo; 2006 [http://www.helsedirektoratet.no/vp/multimedia/archive/00010/IS1292_E_10745a.pdf], IS $1292 \mathrm{E}$.

16. The Norwegian Ministyr of Health and Care Services: The Coordination Reform. Proper treatment - at the right place and right time. Oslo; 2009, St.meld. nr. 47(2008-2009).

17. Andersson H, Ådnanes M: Fastlegen som aktør i tiltak for personer med psykiske problemer (The GP as a participant in work for persons with mental health problems). Oslo: SINTEF report A205; 2006.

18. Classification Committee of WONCA: International Classification of Primary Health Care. Second edition - ICPC-2 edn. Oxford: Oxford University Press; 1998

19. de Stampa M, Vedel I, Bergman H, Novella JL, Lapointe L: Fostering participation of general practitioners in integrated health services networks: incentives, barriers, and guidelines. BMC Health Serv Res 2009, 9:48.

20. Thornhill J, Dault M, Clements D: Ready, set... collaborate? The evidence says "go," so what's slowing adoption of inter-professional collaboration in primary healthcare? Healthc Q 2008, 11:14-16.

21. Ijff MA, Huijbregts KM, van Marwijk HW, Beekman AT, Hakkaart-van Roijen L, Rutten FF, Unutzer J, van der Feltz-Cornelis CM: Cost-effectiveness of collaborative care including PST and an antidepressant treatment algorithm for the treatment of major depressive disorder in primary care; a randomised clinical trial. BMC Health Serv Res 2007, 7:34.

22. Vass $M$, Avlund $K$, Siersma V, Hendriksen C: A feasible model for prevention of functional decline in older home-dwelling people-the GP role. A municipality-randomized intervention trial. Family practice 2009, 26:56-64.

23. Gravelle H, Hole AR: The work hours of GPs: survey of English GPs. Br J Gen Pract 2007, 57:96-100.

24. van den Berg MJ, de Bakker DH, Westert GP, van der Zee Groenewegen PP: Do list size and remuneration affect GPs' decisions about how they provide consultations? BMC Health Serv Res 2009, 9:39.

25. Oandasan FC, Lingarda LG, Karima L, Jakubovicza A, Whiteheada D, Millera C, Natalie Kenniea K-L, Reevesa NS: The impact of space and time on interprofessional teamwork in Canadian primary health care settings: implications for health care reform. Primary Health Care Research \& Development 2009, 10:151-162.

26. van den Hombergh P, Kunzi B, Elwyn G, van Doremalen J, Akkermans $R$, Grol R, Wensing M: High workload and job stress are associated with lower practice performance in general practice: an observational study in 239 general practices in the Netherlands. BMC Health Sen Res 2009, 9:118.

27. Grytten J, Sorensen R: Practice variation and physician-specific effects. Journal of health economics 2003, 22:403-418.

28. Norwegian Knowledge Centre for the Health Services: : Sosiodemografiske forskjeller i bruk og adgang til helsetjeneste i Norge - en kunnskapsoppsummering. (Sociodemographic differences in use of and acess to health care in Norway - a litterature review). Oslo; 2007.

29. Dalstra JA, Kunst AE, Borrell C, Breeze E, Cambois E, Costa G, Geurts Jل Lahelma $\mathrm{E}$, Van Oyen H, Rasmussen NK, et al: Socioeconomic differences in the prevalence of common chronic diseases: an overview of eight European countries. Int J Epidemiol 2005, 34:316-326.

30. Gjesdal S, Svedberg P, Hagberg J, Alexanderson K: Mortality among disability pensioners in Norway and Sweden 1990-96: comparative prospective cohort study. Scand J Public Health 2009, 37:168-175.

31. Naper SO: All-cause and cause-specific mortality of social assistance recipients in Norway: a register-based follow-up study. Scand J Public Health 2009, 37:820-825.

32. van Steenbergen-Weijenburg KM, van der Feltz-Cornelis CM, Horn EK, van Marwijk HW, Beekman AT, Rutten FF, Hakkaart-van Roijen L: Cost-effectiveness of collaborative care for the treatment of major depressive disorder in primary care. A systematic review. BMC Health Sen Res 2010, 10:19. 
33. Iliffe S: From general practice to primary care: the industrialization of family medicine. Oxford: Oxford University Press; 2008.

34. Brage S, Bentsen BG, Bjerkedal T, Nygard JF, Tellnes G: ICPC as a standard classification in Norway. Family practice 1996, 13:391-396.

35. Britt $H$, Angelis $M$, Harris $E$ : The reliability and validity of doctor-recorded morbidity data in active data collection systems. Scandinavian journal of primary health care 1998, 16:50-55.

36. World Health Organisation: Closing the gap in a generation: Health equity through action on the social determinants of health. Geneva; 2008.

37. Grumbach $\mathrm{K}$, Bodenheimer $\mathrm{T}$ : Can health care teams improve primary care practice? JAMA 2004, 291:1246-1251.

38. Lemieux-Charles L, McGuire WL: What Do We Know about Health Care Team Effectiveness? A Review of the Literature. Med Care Res Rev 2006, 63:263-300.

\section{Pre-publication history}

The pre-publication history for this paper can be accessed here: http://www.biomedcentral.com/1472-6963/10/309/prepub

\section{Submit your next manuscript to BioMed Central} and take full advantage of:

- Convenient online submission

- Thorough peer review

- No space constraints or color figure charges

- Immediate publication on acceptance

- Inclusion in PubMed, CAS, Scopus and Google Scholar

- Research which is freely available for redistribution

Submit your manuscript at www.biomedcentral.com/submit
C Biomed Central 